
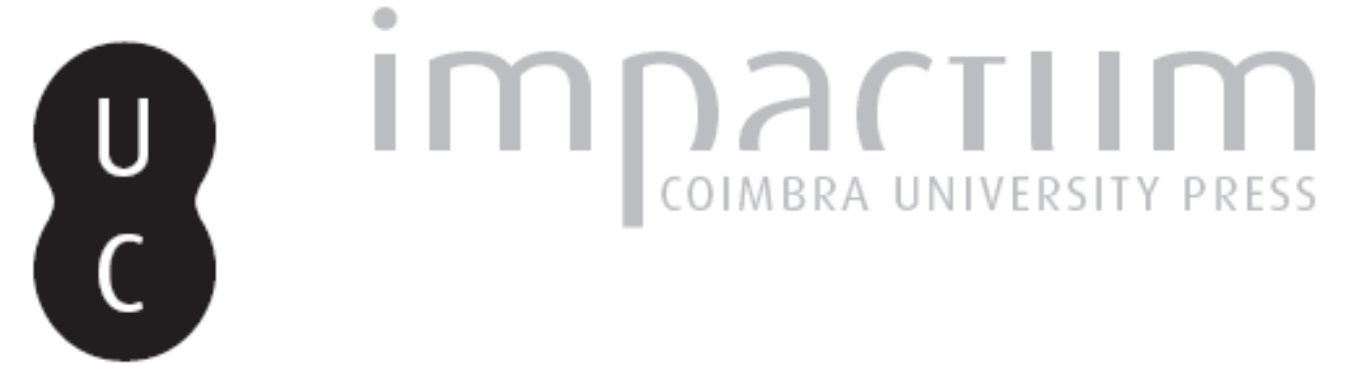

\title{
Relectura de un epígrafe de San Esteban de Gormaz: Soria
}

Autor(es): Gómez-Pantoja, J.

Publicado por: Imprensa da Universidade de Coimbra

URL persistente:

URI:http://hdl.handle.net/10316.2/45465

DOI:

DOI:https://dx.doi.org/10.14195/1647-8657_34_7

Accessed : $\quad$ 26-Apr-2023 11:33:28

A navegação consulta e descarregamento dos títulos inseridos nas Bibliotecas Digitais UC Digitalis, UC Pombalina e UC Impactum, pressupõem a aceitação plena e sem reservas dos Termos e Condições de Uso destas Bibliotecas Digitais, disponíveis em https://digitalis.uc.pt/pt-pt/termos.

Conforme exposto nos referidos Termos e Condições de Uso, o descarregamento de títulos de acesso restrito requer uma licença válida de autorização devendo o utilizador aceder ao(s) documento(s) a partir de um endereço de IP da instituição detentora da supramencionada licença.

Ao utilizador é apenas permitido o descarregamento para uso pessoal, pelo que o emprego do(s) título(s) descarregado(s) para outro fim, designadamente comercial, carece de autorização do respetivo autor ou editor da obra.

Na medida em que todas as obras da UC Digitalis se encontram protegidas pelo Código do Direito de Autor e Direitos Conexos e demais legislação aplicável, toda a cópia, parcial ou total, deste documento, nos casos em que é legalmente admitida, deverá conter ou fazer-se acompanhar por este aviso.

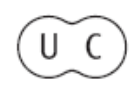


UNIVERSIDADE DE COIMBRA

FACULDADE DE LETRAS

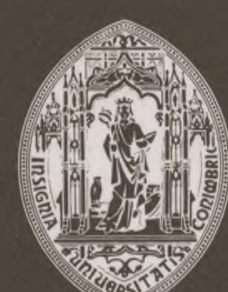

CONIMBRIGA

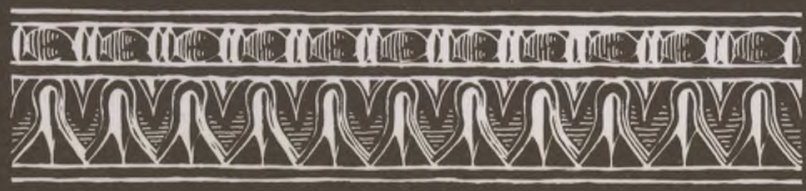

VOLUME XXXIV - 1995

INSTITUTO DE ARQUEOLOGIA 


\section{J. Gómez-Pantoja}

Professor Titular da Universidade de Alcalá de Henares

\section{RELECTURA DE UN EPIGRAFE}

DE SAN ESTEBAN DE GORMAZ, SORIA

"Conimbriga" XXXIV (1995) p. 185-189

RESUMEN: Esta breve nota corrige la lectura de un epitafio latino descubierto en San Esteban de Gormaz, Soria. Se ignora el paradero de la inscripción y el texto sólo era conocido por el relato de un testigo de 1931 ; ahora, la nueva versión ha sido posible gracias al hallazgo en el archivo personal de F. Fita de una carta de 1912 conteniendo una transcripción independiente.

SUMMARY: This brief note offers a corrected reading of a Roman tombstone from San Esteban de Gomaz, Soria, Spain. Previous editions of the text resulted in anomalous reading and no checking was possible since stone is lost. My note benefits from a letter of 1912 discovered in F. Fita's files and containing a better rendering of the inscription. 
(Página deixada propositadamente em branco) 


\title{
RELECTURA DE UN EPÍGRAFE DE SAN ESTEBAN DE GORMAZ, SORIA
}

La lápida, hoy desaparecida, sólo se conocía por lo que de ella dice un culto turista de hace sesenta años (O- Esa noticia proporciona las dimensiones del epígrafe, su situación entonces y una transcripción del texto difícilmente inteligible y que más parece una interpolación:

\author{
CALNV S. A. EMILI \\ AELITA NIONIS F. \\ ANN. XX CANTA \\ BRA SOROR QUI \\ NA SOROR H. S. \\ FLORVS TECVLA \\ AVSTICA D. S. P. E C.
}

Estudiosos posteriores han resaltado las anomalías de la tradición pero ante la imposible autopsia de una pieza en paradero desconocido, sólo cabia normalizar lo recibido. Como esos intentos no han resultado muy afortunados $\left({ }^{2}\right)$, hace apenas un año propuse la siguiente relectura del epígrafe ( $\left.{ }^{3}\right)$ :

(') P. Artigas, "Por tierras de gesta. San Esteban de Gormaz", BSEE, 40, 1932, p. 41.

(2) C. García Merino, "Un olvidado núcleo de población hispanorromano: San Esteaban de Gormaz", Hispania Antiqua 7, 1977, pp. 185-7: Calnu(s) s(ervus) Aemilio Litanionis flilius) an(norum) XX Cantabra soror, Quinta soror h(ic) s(unt). Florus, Tecula (?) Rustica d(e) s(ua) p(ecunia) f(aciendum) c(uraverunt). Por su parte, A. Jimeno, Epigrafia romana de la provincia de Soria, Soria, 1980, p. 122, propuso Calnus (?) Aemilia Elitanionis f(ilia) ann... etc, y prefirió entender el nombre de una de las dedicantes como Tecula Austica.

(3) J. Gómez-Pantoja, "Viejas piedras, nuevas lecturas. Inscripciones latinas de San Esteban de Gormaz, Soria", en C. Saéz y J. Gómez-Pantoja, Las diferentes historias de letrados y analfabetos, Alcalá 1994, pp. 214-5. 
Calvus • Aemili / $\propto$ - Litanionis - f(ilius) • / ann(orum) • XX Canta/bra soror QuiVfita soror h(ic) - s(epulti) [s(unt)] • / Florus, fecula, / Rustica d(e) $s($ ua $) \cdot p($ ecunia $) \cdot f($ aciendum $) \cdot c($ uraverunt $)$

Recientemente, sin embargo, entre la correspondencia inédita de F. Fita $\left({ }^{4}\right)$, he encontrado un memorial del 7 de agosto de 1912 enviado por Andrés Serrano, Jefe de Telégrafos de San Esteban de Gormaz. Entre otras noticias de diversa consideración sobre las antigüedades del lugar, aparecen los párrafos que transcribo literalmente:

"Hará unos diez años se extrajo de la muralla de tierra del Castillo en la que servía de cimiento una magnifica losa con la inscripción seguiente:

\section{CAIVS AEMILI \\ AE LITANIONIS.F \\ ANN XX CANTA \\ BRA SORO ROVI \\ NA SOR\&R.HSS \\ TLORV// TECVLA \\ AVSTICA OSIEO}

Está colocada en el pavimento del primer tramo de la escalera de la casa de D. Faustino Espinar, vecino de esta villa. Mandé que la copiaran con toda fidelidad y no sé si habrán alterado alguna letra.

Además de los datos sobre el lugar de aparición de la pieza, la carta del ilustrado y sensible telegrafista permite, en mi opinión, recuperar con bastante seguridad el texto original del epígrafe, que no sería otro que:

Calus • Aemili/ce - Litanionis - f(ilius) - / ann(orum) • XX. Canta/bra soror, OvP/na soro h(ic) • s(epulti) s(unt) $\bullet / F^{\wedge}$ loru[s], Tegula, /Mustica ${ }^{v} d\left(\right.$ ep $^{\bullet} \wedge\left(\right.$ uap $^{\wedge}$ piecuniaf fiaciendumj $\bullet{ }^{r}$ c(uraveruntjl

$\left(^{4}\right) \quad$ Este trabajo ha sido posible gracias a la gentileza de Luis García Iglesias, que me introdujo en el archivo Fita y obtuvo para mí la autorización de los PP. Jesuitas para publicarlo. 
Calus es mejor que el irregular Caius y aunque en casos así no puede descartarse la haplología de Calvus, nótese que el mismo nombre aparece también en un reciente hallazgo de Villamesías, Cáceres $\left({ }^{5}\right)$. Sobre Litanion, el testimonio de Serrano ratifica lo avanzado en mi artículo y constituye una solución más lógica que el aventurado Elita de Abascal ( $\left.{ }^{6}\right)$. El raro Ovina está, según parece, documentado en una inscripción de Cárquere (Resende, Viseu) ( ${ }^{7}$ ) y no resulta un forzamiento excesivo de la tradición corregir Tegula por Tecuta: en realidad así lo apunté con dudas por falta de paralelos que, en realidad, sí parece haber; $\left(^{8}\right)$ y aunque no existiesen, nada obsta a un nombre de esas características, especialmente en el ambiente rural o servil del que parece proceder la inscripción. Finalmente, y a salvo de mejor juicio, Rustica es una opción más explicable y satisfactoria que el extraño Austica de Serrano y Artigas.

$\left.{ }^{5}\right)$ J. L. Gamallo y H. Gimeno "Inscripciones del norte y suroeste de la provincia de Cáceres: revisión y nuevas aportaciones", CUPAUAM 17, 1990, p. 284 y el comentario de A. U. Stylow, HEp 4, 1994, 257.

$\left(^{6}\right)$ J. M. Abascal, Los nombres personales en las inscripciones latinas de Hispania, Murcia 1994, p. 351.

(7) HAE 244.

(8) H. Solin y O. Salomies, Repertorium nominum gentilium et cognominum Latinorum, Hildesheim 1988, p. 410. 